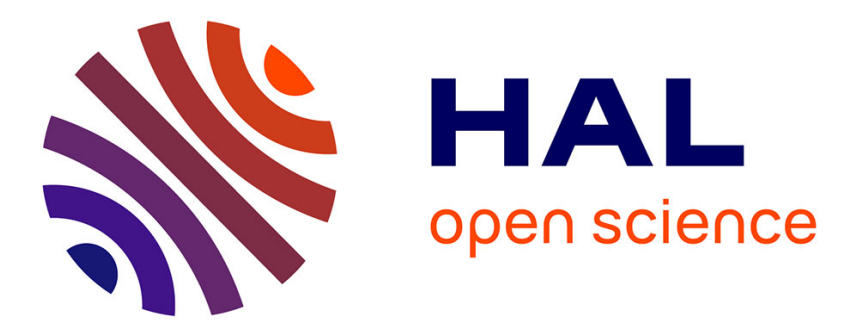

\title{
Learning to classify materials using Mueller imaging polarimetry
}

\author{
Yvain Quéau, Florian Leporcq, Alexis Lechervy, Ayman Alfalou
}

\section{To cite this version:}

Yvain Quéau, Florian Leporcq, Alexis Lechervy, Ayman Alfalou. Learning to classify materials using Mueller imaging polarimetry. Fourteenth International Conference on Quality Control by Artificial Vision (QCAV), May 2019, Mulhouse, France. pp.111720Z, 10.1117/12.2516351 • hal-02088951v2

\section{HAL Id: hal-02088951 https://hal.science/hal-02088951v2}

Submitted on 3 May 2019

HAL is a multi-disciplinary open access archive for the deposit and dissemination of scientific research documents, whether they are published or not. The documents may come from teaching and research institutions in France or abroad, or from public or private research centers.
L'archive ouverte pluridisciplinaire HAL, est destinée au dépôt et à la diffusion de documents scientifiques de niveau recherche, publiés ou non, émanant des établissements d'enseignement et de recherche français ou étrangers, des laboratoires publics ou privés. 


\title{
Learning to classify materials using Mueller imaging polarimetry
}

\author{
Yvain Quéau $^{1, *}$, Florian Leporcq ${ }^{2}$, Alexis Lechervy ${ }^{1}$, Ayman $^{\text {Alfalou }}{ }^{3}$ \\ ${ }^{1}$ GREYC, UMR CNRS 6072, Université de Caen Normandie, Caen, France \\ ${ }^{2}$ INP-ENSEEIHT, Université Fédérale Toulouse-Midi-Pyrénées, Toulouse, France \\ ${ }^{3}$ L@bISEN Yncrea-Ouest, ISEN Brest, Brest, France
}

\begin{abstract}
This study investigates the combination of Mueller imaging polarimetry with machine learning for the automated optical classification of raw materials. It shows that standard image classification techniques based on support vector machines or deep neural networks can readily be applied to polarimetric data extracted from Mueller matrix measurements. The feasibility of such an approach is empirically demonstrated through the classification of multispectral depolarization images of real-world materials (banana, wood and foam samples).
\end{abstract}

Keywords: Material classification, polarimetry, Mueller imaging, Machine Learning, Deep Neural Networks.

\section{INTRODUCTION}

Mueller polarimetry ${ }^{1}$ allows one to measure the polarization properties of a medium. Such measurements, coupled with machine learning techniques, have found successful applications in e.g., biomedical diagnosis,, 2$] 3$ car detection ${ }^{4}$ or material classification $\sqrt[56]{6}$ While the classification of image patches into different material categories is a classic image processing problem ${ }^{7}$ for which various multispectral ${ }^{8}$ photometric $^{9}$ or polarizationbased 10 11 solutions exist, very few works in this direction considered active polarimetry, although Zallat et al. had already demonstrated in $2003^{5}$ that it could allow the automated classification of an image into different clusters corresponding to different materials. More recently, Vaughn et al. showed in ${ }^{6}$ that materials could be classified through their Mueller matrices using support vector machines $\left(\mathrm{SVM}^{12}\right)$, but they classified single Mueller matrices and not spatially-varying fields of Mueller matrices, as would be the case in polarimetric imaging. ${ }^{5}$

On the other hand, we believe that the spatially-varying properties of polarization, i.e., the texture, may be of fundamental importance for characterizing non-uniform materials. Therefore, we revisit the problem of material classification using active polarimetric imaging, in view of the recent developments in the field of image classification due to the advent of deep neural networks. Our aim is to show that state-of-the-art image classification techniques such as ${ }^{13}$ can readily be applied to polarimetric images, and that image classification benefits from the introduction of polarization data.

Consider for instance the images in Figure 1. which depict three small samples of banana, wood and foam, imaged at 510, 580 and $630 \mathrm{~nm}$ using a multispectral camera. The intensity images of these samples appear only slightly different, and therefore classification of these images will be rather difficult. On the other hand, turning these images into polarimetric images (in this example, we extracted the depolarization index from Mueller matrix fields of the same samples) reveals structures which are not visible in the intensity images. Therefore, polarization images seem to much better characterize materials in comparison with intensity images, and we shall see that they can readily be plugged into existing image classification frameworks.

The methodology we followed for constructing a database of polarization images of different materials is presented in Section 2. In Section 3, we report preliminary results which tend to indicate the soundness of plugging polarimetric images into image classification frameworks. Eventually, Section 4 draws our conclusions and suggests several future research directions.

\footnotetext{
* yvain.queau@ensicaen.fr
} 


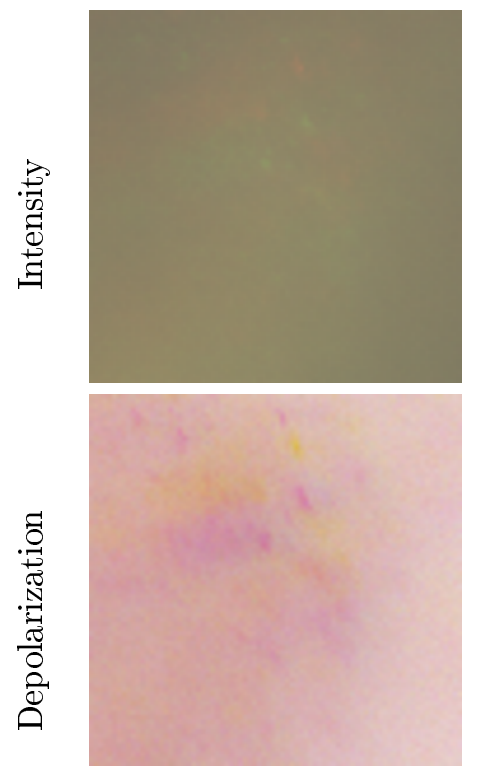

Banana
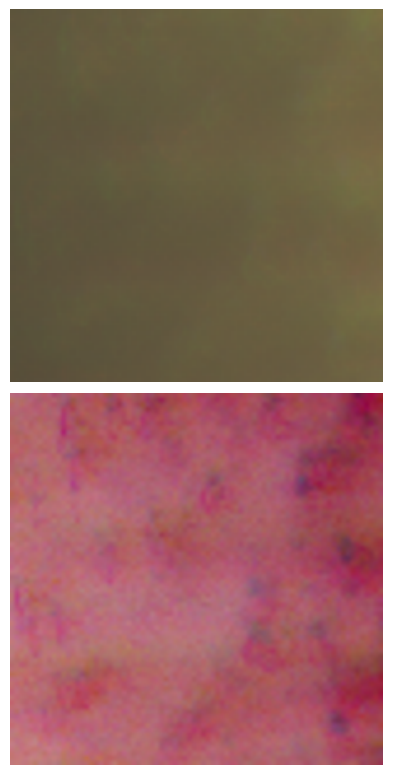

Wood
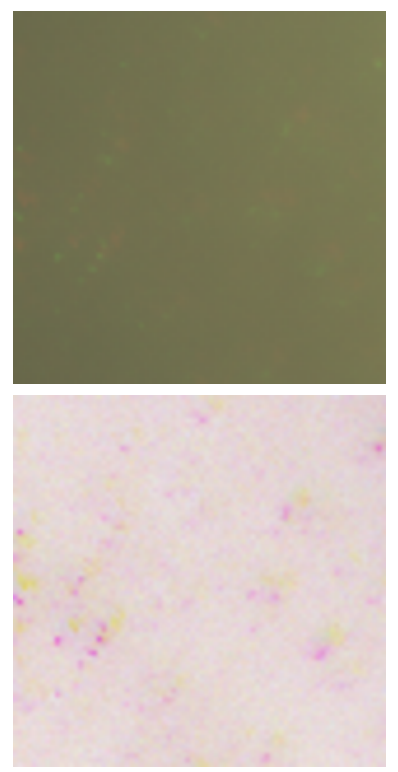

Foam

Figure 1. Comparison between intensity and depolarization images of banana, wood and foam samples (each sample is approximately $1 \mathrm{~cm}^{2}$ large). In both rows, the blue, green and red channels encode information measured, respectively, at 510, 580 and $630 \mathrm{~nm}$ using a multispectral camera. While it is very difficult to distinguish between the three samples using intensity, depolarization reveals the underlying structures of the matter and is thus more suitable for classification purposes.

\section{ACQUISITION OF POLARIMETRIC DATA}

In order to create a database of polarimetric images, we considered the dual-rotating Mueller imaging polarimeter presented in Figure 2 and described in detail in. ${ }^{14}$ It consists a collimated halogen light source followed by a polarization state generator (a fixed linear polarizer followed by a controllable quaterwave plate), a polarization state analyzer (a controllable quaterwave plate followed by a controllable linear polarizer) and a multispectral camera. The sample to analyze is placed between the polarization state generator and polarization state analyzers. By acquiring a series of images under several predefined polarization angles for the optical elements in the generator and analyzer, the polarization properties of the sample can be estimated.

More formally, let the polarization state of the light entering the generator be represented by the Stokes vector $[\mathrm{I}, \mathrm{Q}, \mathrm{U}, \mathrm{V}]^{\dagger}$, and let the polarization state of the light exiting the analyzer be represented by the Stokes vector $[I, Q, U, V]^{\top}$. Let $A, M$ and $G$ be the $4 \times 4$ Mueller matrices of the analyzer, medium to analyze and generator, respectively. Then, the transformations in the polarization state of light can be represented as follows:

$$
\left[\begin{array}{llll}
I & Q & U & V
\end{array}\right]^{\top}=A M G\left[\begin{array}{llll}
\mathrm{I} & \mathrm{Q} & \mathrm{U} & \mathrm{V}
\end{array}\right]^{\top}
$$

In dual-rotating polarimetry, a non-polarized light source is used (i.e., $\mathrm{Q}=\mathrm{U}=\mathrm{V}=0$ in Eq. (1), the Mueller matrices $A$ and $G$ of the analyzer and generator are controllable, and a photosensitive sensor measures the output intensity $I$. The Mueller matrix $M$ of the medium can then be estimated using linear least-squares, from a series of 64 intensity measurements obtained under known varying states for the analyzer and generator.

As can be seen in Figure 2, Mueller matrices of real-world samples may exhibit both spectrally and spatiallyvaring properties. In order to capture as many information as possible about the imaged material, it is thus interesting to measure not just one Mueller matrix, but rather a set of Mueller matrix fields representing both the spatial and spectral variations of the material's polarization properties. These properties can then be represented in a more compact form by turning the Mueller matrix fields into various physically-meaningful quantities such as diattenuation, retardance, polarizance or depolarization. In this study we focus for simplicity on depolarizing materials, and thus we turn the estimated Mueller matrix fields into depolarization images (cf. Figure 3). For 


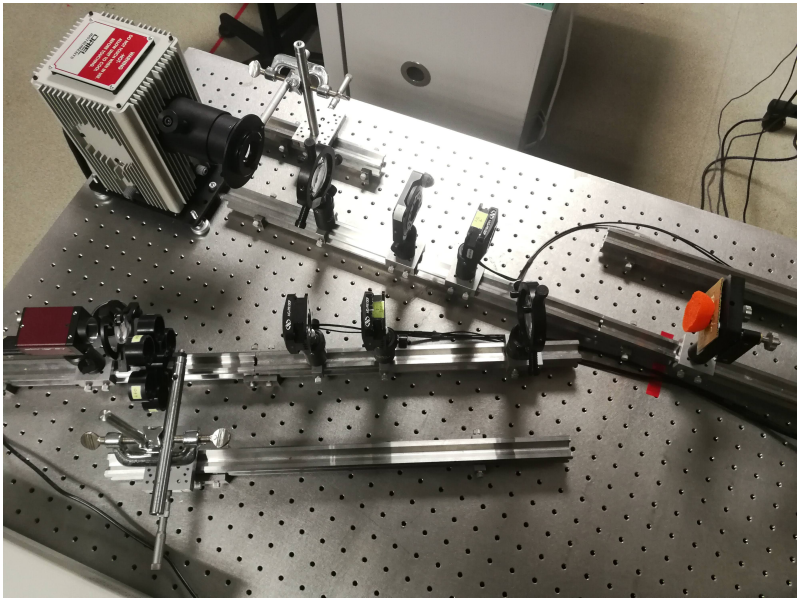

Mueller imaging polarimeter
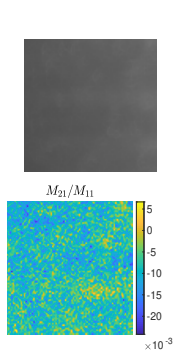

$M_{31} / M_{1}$

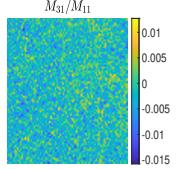

$M_{41} / M_{11}$

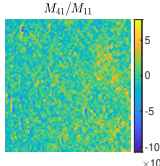

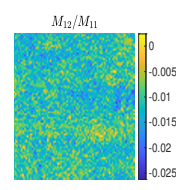

$M_{22} / M_{11}$

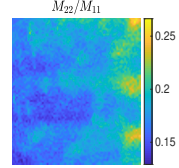

$M_{32} / M_{11}$

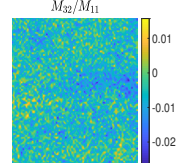

$M_{42} / M_{11}$

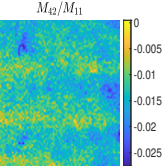

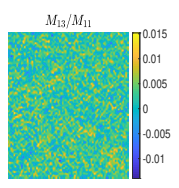
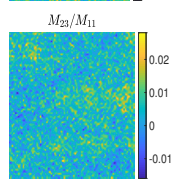

$M_{33} / M_{11}$

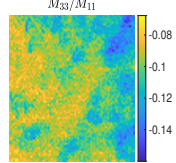

$M_{43} / M_{11}$

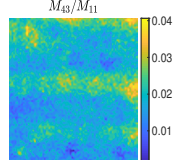

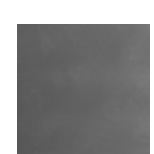

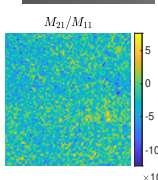

$M_{31} / M_{11}$

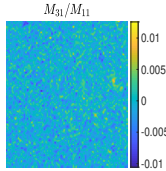

$M_{41} / M_{11}$

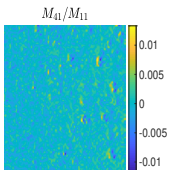

Mueller matrix field at $510 \mathrm{~nm}$

$M_{22} / M_{11}$

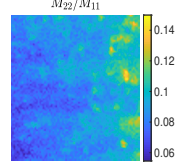

$M_{32} / M_{11}$

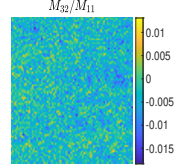

$M_{23} / M_{11}$

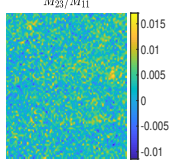

$M_{33} / M_{11}$

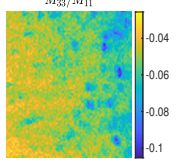

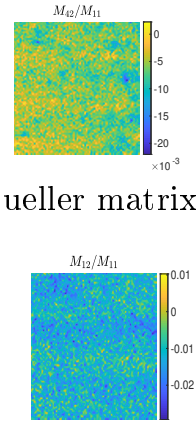

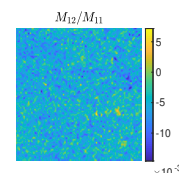

$M_{22} / M_{11}$

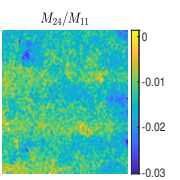

$M_{14} / M_{11}$

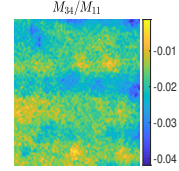

$M_{44} / M_{11}$

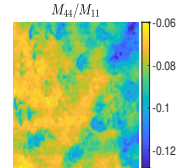

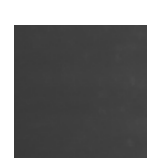

$M_{22} / M_{11}$

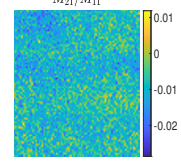

$M_{31} / M_{11}$

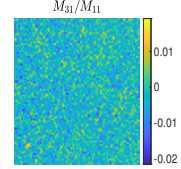

$M_{41} / M_{11}$

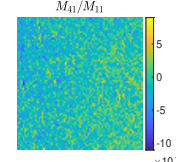

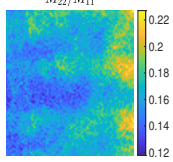

$M_{32} / M_{11}$

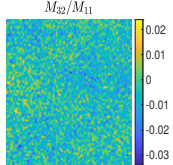

$M_{42} / M_{11}$

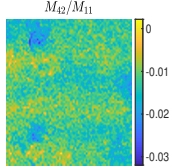

Mueller matrix field at $580 \mathrm{~nm}$
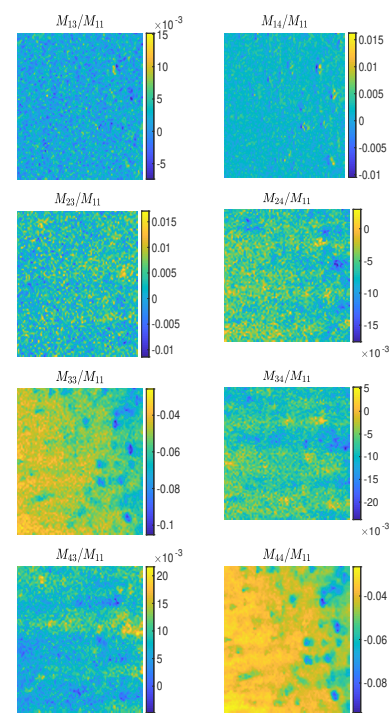

Mueller matrix field at $630 \mathrm{~nm}$

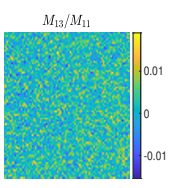

$M_{23} / M_{11}$

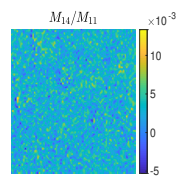

$M_{24} / M_{11}$

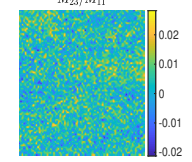

$M_{33} / M_{11}$

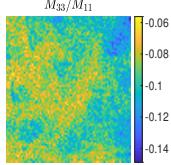

$M_{43} / M_{11}$
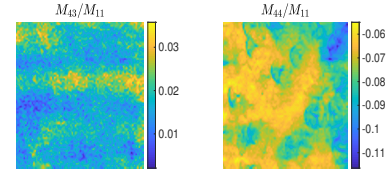

Figure 2. Top, left: Mueller imaging polarimeter used in our experiments (top left is a collimated light source followed by a polarization state generator, bottom left is the multispectral sensor preceded by a polarization state analyzer, right is the medium to be analyzed). This polarimeter is used to obtain 2D Mueller matrix fields characterizing the optical properties of the medium at three different wavelengths (in each case, the top-left image shows one intensity image of the medium, and the others show the Mueller matrix components normalized by the first one). The three Mueller matrix fields are then turned into depolarization images (cf. Figure 33. The Mueller matrix fields shown in this example are those from the wooden sample in the middle column of Figure 1 .

one particular pixel and one particular wavelength, we define depolarization as in Equation (47) in, 1,1 such that it varies from 0 for a nondepolarizing Mueller matrix to 1 for an ideal depolarizer:

$$
\text { Depolarization }=1-\frac{\sqrt{\sum_{i=1}^{4} \sum_{j=1}^{4} \frac{M_{i j}{ }^{2}}{M_{11}{ }^{2}}-1}}{\sqrt{3}} .
$$

The multispectral depolarization images obtained this way can then be classified using machine learning techniques, as discussed in the next section. 


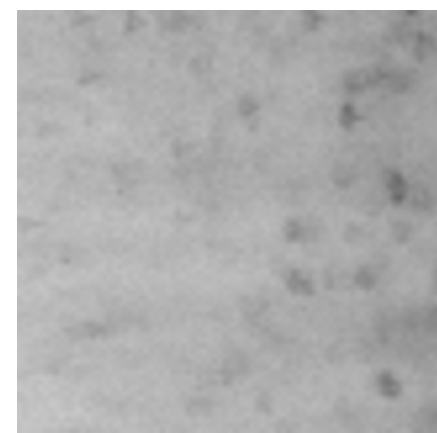

$510 \mathrm{~nm}$

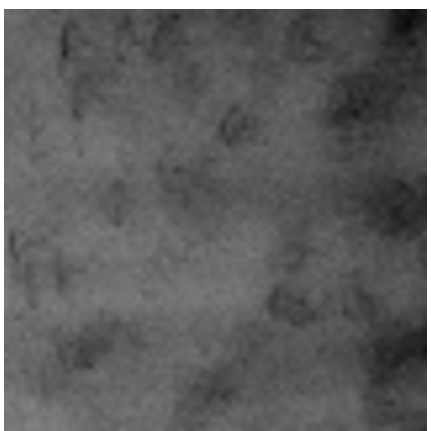

$580 \mathrm{~nm}$

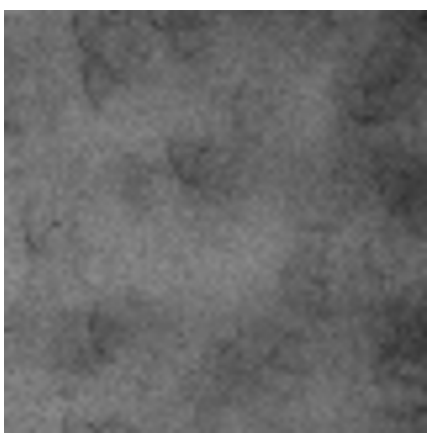

$630 \mathrm{~nm}$

Figure 3. Depolarization images corresponding to the three Mueller matrix fields shown in Figure 2 These three gray level images are then combined into RGB images such as the ones in the second row of Figure 1 and used for classification purposes.

\section{CLASSIFYING MULTISPECTRAL DEPOLARIZATION IMAGES}

Next, we assess the ability of machine learning techniques to classify depolarization images constructed as discussed in the previous section. The point which we aim at making is that such frameworks, which are usually designed for the classification of natural RGB images, can handle polarization clues without particular tedious tuning; and that classification of materials based on polarization is competitive with the standard approach based on RGB intensities.

In order to provide a proof of concept for these claims, we constructed a database of intensity and depolarization images for three different classes: banana, wood and foam. At small-scale (each image pictures an area of approximately $1 \mathrm{~cm}^{2}$ ), the intensities of these three samples look more or less alike and there seems to be very few information to extract for classification. However, the three classes exhibit very different spatially-varying polarization properties: there is much more salient features to observe in the depolarization images and thus one would expect that such images could be used in a standard image classifier.

We constructed an annotated training set consisting of 100 intensity and depolarization images per class. Both were constructed by imaging the samples at 510, 580 and $630 \mathrm{~nm}$, and assigning the resulting measurements to the blue, red and green channels of RGB images of size $224 \times 224$. Another set of 50 test images per class, different from the training images, was then acquired. Exemplar images from the training and test sets are shown in Figure 4

We then trained two standard image classifiers on the training set, and evaluated their performance on the test set. First, we considered the image classifier from Matlab's computer vision toolbox, which is a support vector machine (SVM) classifier relying on bag of SURF features $\frac{15}{15}$ Since our purpose is to question whether or not such frameworks can readily be used by nonspecialists in machine learning, we refrained ourselves from tuning any parameter of the classifier, and used all the default ones. The results shown in the first row of Figure 5 indicate that the performances of this naive approach on intensity images are not really satisfactory. Obviously, better performances could be obtained through parameter tweaking, but it is much more interesting to observe that even without any tuning, performances drastically improve when feeding the classifier with depolarization images instead of intensity images. This tends to indicate that the underlying information is both easier to extract and more discriminative.

Another alternative to improve the results is to resort to a state-of-the-art convolutional neural network $(\mathrm{CNN})$ for the classification, instead of the SVM. We thus also evaluated the performances of a Resnet 18 network ${ }^{13}$ for this task. Since training such a network requires optimizing over several millions of parameters, and we have at our disposal only a few hundreds of images, we carried out data augmentation of the training set (rotation, translation and scaling of images) and initialized the parameters by pretraining on the ImageNet database. ${ }^{16}$ The results shown in the second row of Figure 5 indicate once again that the classifier performs much better on polarization-aware images than on plain intensity images. 

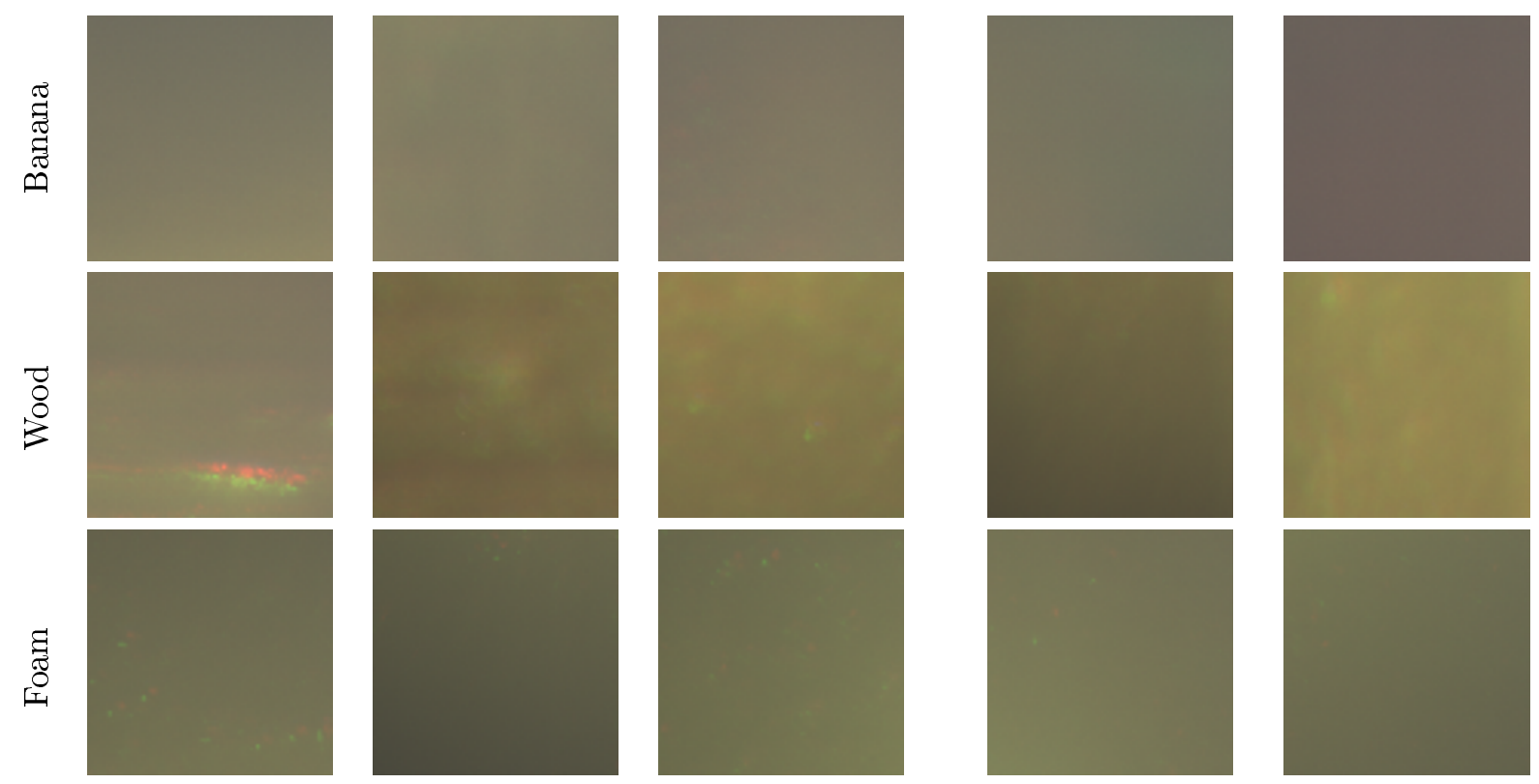

Intensity samples from the training set

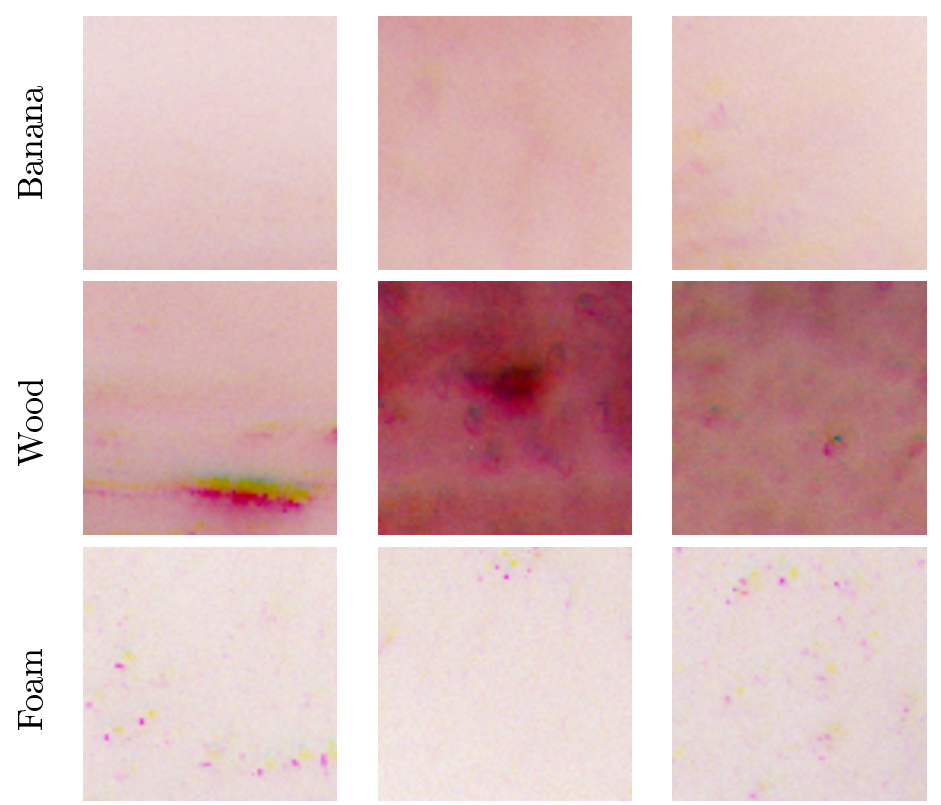

Depolarization samples from the training set

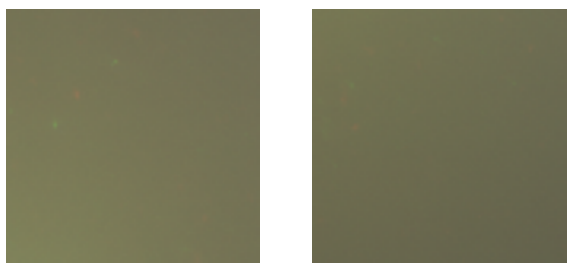

Intensity samples from the testing set
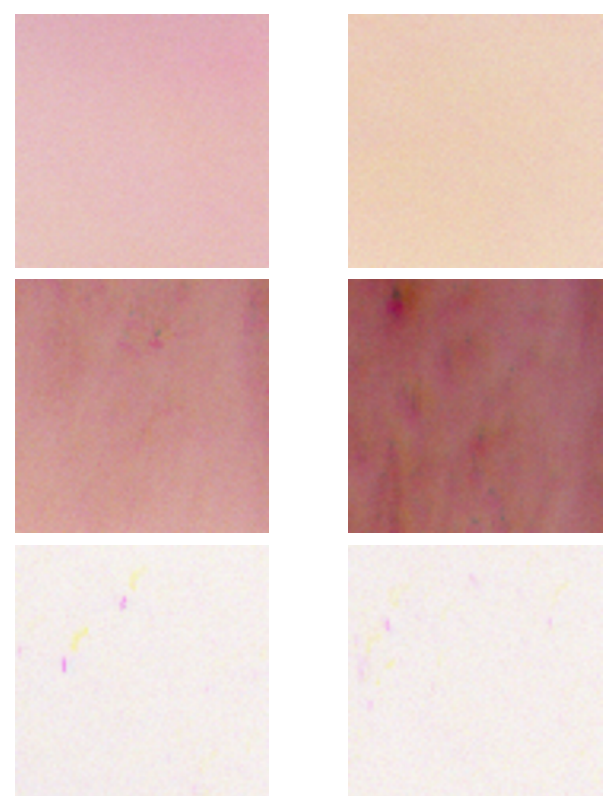

Depolarization samples from the testing set

Figure 4. Real-world banana, wood and foam samples used for classification. Top: multispectral intensity images, bottom: corresponding depolarization images. Each training set contains 100 images, and each testing set contains 50 images which are not present in the training sets.

We emphasize the simplicity of both experiments, which consist simply in replacing the input to existing image classification architecture from plain images to polarization-aware ones, without any parameter tuning. Obviously, even better performances would be expected by more appropriately tuning the parameters and, most importantly, enlarging the training database. But, the fact that existing architectures are readily applicable to Mueller imaging polarimetry data, and that considering such data seems to yield very reasonable classification results, already highlights the potential of the combination between polarimetry and machine learning. 


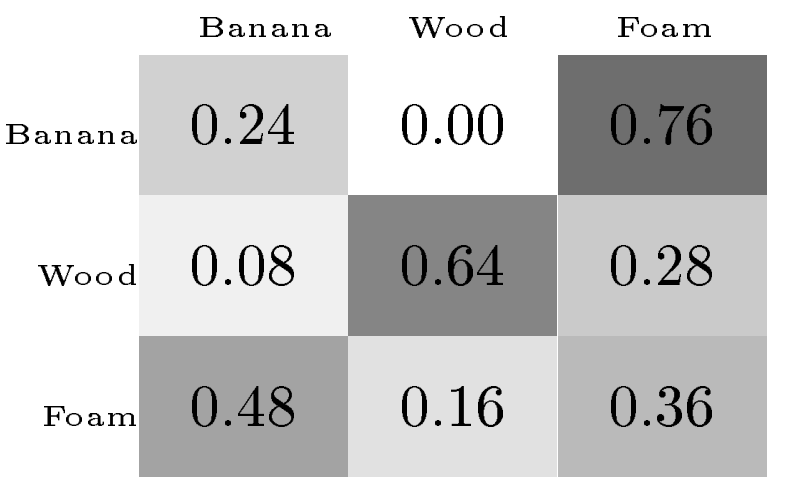

SVM on intensity (avg. accuracy: 0.41)

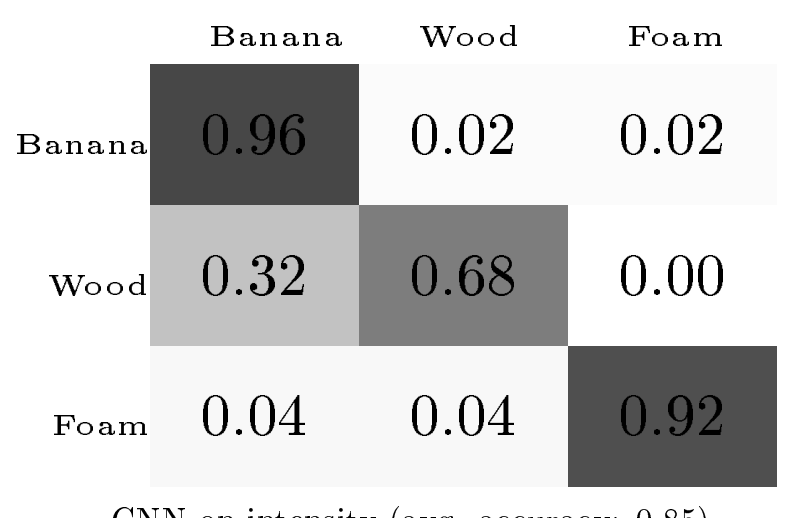

CNN on intensity (avg. accuracy: 0.85)

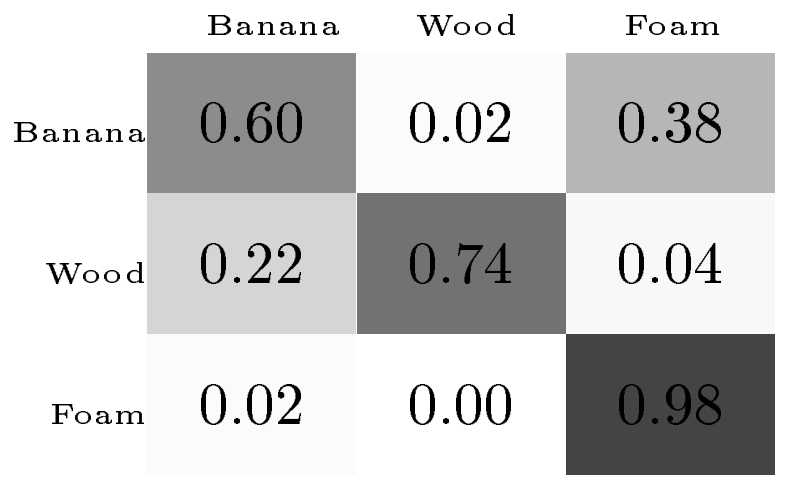

SVM on depolarization (avg. accuracy: 0.77)

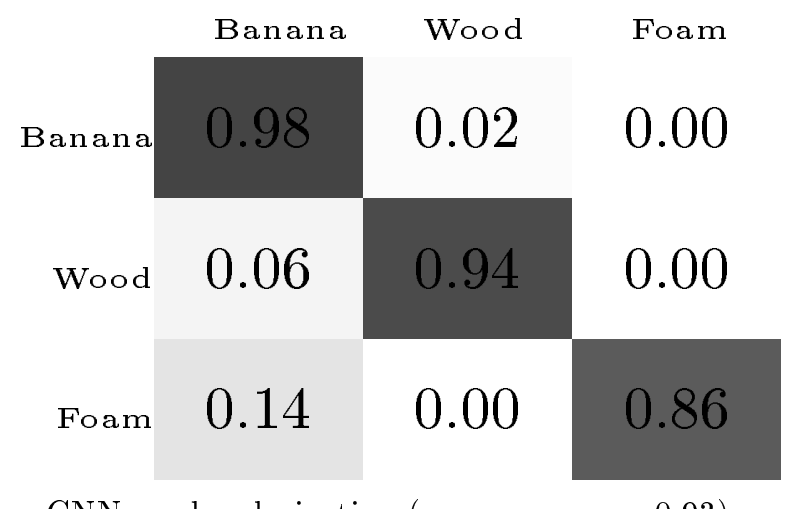

CNN on depolarization (avg. accuracy: 0.93)

Figure 5. Confusion matrices obtained for the classification of real-world banana, wood and foam samples, using Matlab's builtin support vector machine (SVM) classifie ${ }^{[15]}$ with default parameters (top) or a state-of-the-art convolutional neural network $(\mathrm{CNN})^{\sqrt{13}}$ whose parameters are initialized by pretraining on the ImageNet database ${ }^{[16}$ (bottom), on intensity (left) and depolarization (right) images. In each table, the columns show the predicted labels, the rows show the true labels and the numbers indicate the percentage of classifications. Considering depolarization instead of intensity improves the results in both cases: standard image classification tools can thus readily be applied to active polarimetric imaging systems and classification seems to benefit from the use of polarimetry-aware data.

\section{CONCLUSION AND PERSPECTIVES}

We investigated the combination of machine learning techniques and active polarimetry for the purpose of optical classification of heterogeneous materials. We showed that existing image classification techniques could readily be employed for classifying depolarization images constructed by multispectral Muller imaging polarimetry. Classification experiments using support vector machines and convolutional neural networks were carried out on real-world samples, and indicated the potential of polarimetry-aware classification over plain intensity-based approaches. In the future, we plan to further explore the potential of the proposed approach by directly classifying multispectral Mueller matrix fields instead of depolarization images. This represents a nontrivial task: for the moment each depolarization image consists of one "graylevel" image per wavelength, and thus considering three wavelengths we could obtain RGB-like images which can be plugged into existing frameworks. But, using the full Mueller matrix field would result in 16-dimensional images per wavelength, and thus the architecture of the network might need to be adapted. 


\section{REFERENCES}

[1] Chipman, R. A., "Polarimetry," in [Handbook of optics, Volume II], Bass, M., DeCusatis, C., Enoch, J., Lakshminarayanan, V., Li, G., Macdonald, C., Mahajan, V., and Van Stryland, E., eds., ch. 22, McGrawHill, Inc. (2009).

[2] Ahmad, I., Ahmad, M., Khan, K., Ashraf, S., Ahmad, S., and Ikram, M., "Ex vivo characterization of normal and adenocarcinoma colon samples by Mueller matrix polarimetry," Journal of Biomedical Optics 20(5), $056012(2015)$.

[3] Qi, J. and Elson, D. S., "Mueller polarimetric imaging for surgical and diagnostic applications: a review," Journal of Biophotonics 10(8), 950-982 (2017).

[4] Fan, W., Ainouz, S., Meriaudeau, F., and Bensrhair, A., "Polarization-Based Car Detection," in [Proceedings of the IEEE International Conference on Image Processing (ICIP)], 3069-3073, IEEE (2018).

[5] Zallat, J., Grabbling, P., and Takakura, Y., "Using polarimetric imaging for material classification," in [Proceedings of the IEEE International Conference on Image Processing (ICIP)], 2, II-827 (2003).

[6] Vaughn, I. J., Hoover, B. G., and Tyo, J. S., "Classification using active polarimetry," Proc. SPIE $\mathbf{8 3 6 4}$ (2012).

[7] Varma, M. and Zisserman, A., "A statistical approach to material classification using image patch exemplars," IEEE Transactions on Pattern Analysis and Machine Intelligence 31(11), 2032-2047 (2009).

[8] Deborah, H., Richard, N., and Hardeberg, J. Y., "Application of spectral statistics to spectral texture discrimination," in [Proceedings of the IEEE Colour and Visual Computing Symposium (CVCS)], 1-6 (2018).

[9] Kampouris, C., Zafeiriou, S., Ghosh, A., and Malassiotis, S., "Fine-grained material classification using micro-geometry and reflectance," in [Proceedings of the European Conference on Computer Vision], 778-792 (2016).

[10] Chen, H. and Wolff, L. B., "Polarization phase-based method for material classification in computer vision," International Journal of Computer Vision 28(1), 73-83 (1998).

[11] Tominaga, S. and Yamamoto, T., "Metal-dielectric object classification by polarization degree map," in [Proceedings of the IEEE International Conference on Pattern Recognition], (2008).

[12] Boser, B. E., Guyon, I. M., and Vapnik, V. N., "A training algorithm for optimal margin classifiers," in [Proceedings of the fifth annual workshop on Computational learning theory], 144-152 (1992).

[13] He, K., Zhang, X., Ren, S., and Sun, J., "Deep residual learning for image recognition," in [Proceedings of the IEEE conference on Computer Vision and Pattern Recognition], 770-778 (2016).

[14] Quéau, Y., Leporcq, F., and Alfalou, A., "Design and simplified calibration of a Mueller imaging polarimeter for material classification," Optics Letters 43(20), 4941-4944 (2018).

[15] Csurka, G., Dance, C., Fan, L., Willamowski, J., and Bray, C., "Visual categorization with bags of keypoints," in [Proceedings of the ECCV Workshop on statistical learning in computer vision], 1(1-22), 1-2 (2004).

[16] Deng, J., Dong, W., Socher, R., Li, L.-J., Li, K., and Fei-Fei, L., "Imagenet: A large-scale hierarchical image database," in [Proceedings of the IEEE conference on Computer Vision and Pattern Recognition], 248-255 (2009). 